\title{
PREVALENCE OF MICROALBUMINURIA AND PREDICTIVE FACTORS IN A GROUP OF ALBANIAN DIABETIC ADULTS
}

\section{E. Spahiu ${ }^{1}$, F. Toti ${ }^{1}$, A. Doko ${ }^{2}$, M. Carcani ${ }^{1}$,L. Bruka ${ }^{1}$, M. Ismaili ${ }^{1}$, A. Shehu ${ }^{3}$ \\ 1. Service of Endocrinology \& Metabolic diseases University Hospital Center "Mother Theresa" Tirana/ALBANIA \\ 2. Service of Cardiology University Hospital Center "Mother Theresa" Tirana/ALBANIA \\ 3. "Intermedica" Laboratory Tirana/ALBANIA

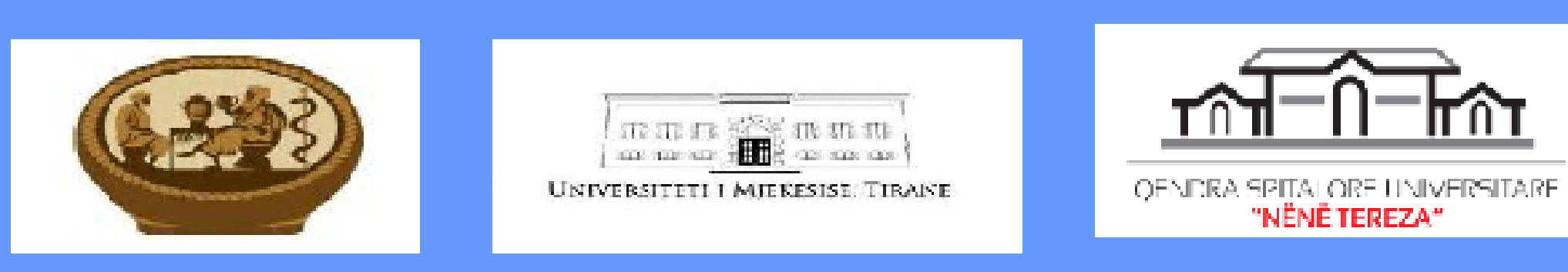

Diabetic nephropathy is one of the most prevalent and dangerous chronic complication of diabetes mellitus.

Its end-stage, renal chronic failure is the most important cause of premature death in diabetic patients. Microalbuminuria(MA), one of the first signs of Diabetic nephropathy, may be present from the moment of diagnosis in type 2 diabetes.

The aim of our study was to determine the prevalence of microalbuminuria and to identify predictor factors in a group of albanian diabetic adult patients.

\section{Methods:}

Diabetic patients selected randomly, assigned by hospital admissions in Tirana University Hospital Center, Durres and Fieri regional hospitals.

Biochemical examinations, anthropometric measurements, and measurement of microalbuminuria by DCA 2000 in an urine spot, was performed.

Microalbuminuria was considered positive if $\geq 20 \mathrm{mg}$ or albumine/creatinine report $\geq 25$.

\section{Results:}
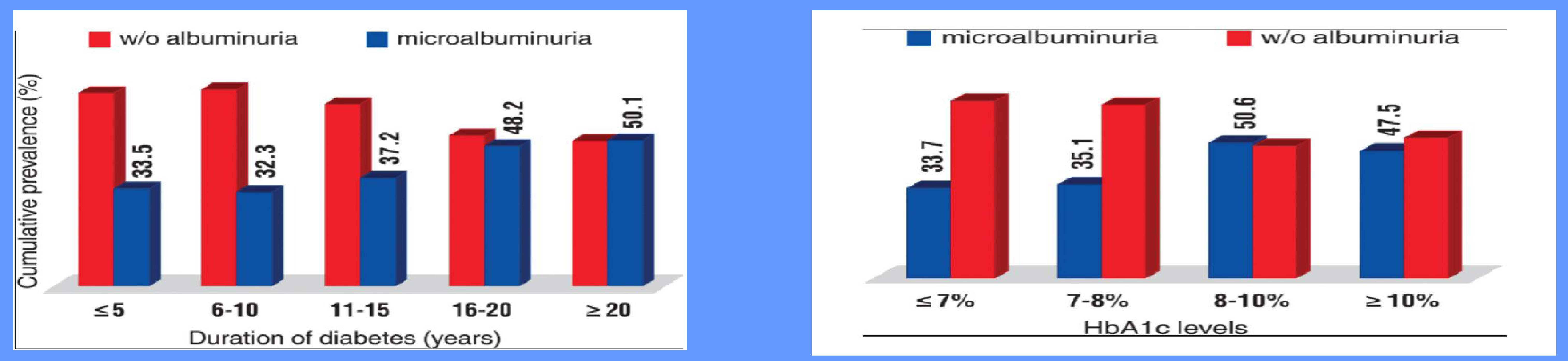

\begin{tabular}{|c|c|c|c|c|c|c|c|c|}
\hline \multicolumn{4}{|c|}{$\begin{array}{l}\text { Table } 1 \text { : Univariate analysis revealed that the risk factors associated with microalbuminuria were duration of diabetes, waist } \\
\text { circumference in females, HbAlc and systolic blood pressure. }\end{array}$} & \multicolumn{5}{|c|}{ Table 2: Multiple regression analysis using miccoalbuminiuria as the dependent varable. } \\
\hline Gender & $\frac{\text { Odds ratio }}{1.334}$ & $\frac{95 \% \text { confidence interval }}{0.958-3.243}$ & $\frac{p \text {-value }}{0.152}$ & & \multicolumn{2}{|c|}{ Males } & \multicolumn{2}{|c|}{ Females } \\
\hline $\mathrm{HbAlc}$ & 2.011 & $1.596-4.253$ & $<0.005$ & & Odds ratio & $95 \% \mathrm{CI}$ & Odds ratio & $95 \% \mathrm{CI}$ \\
\hline $\begin{array}{l}\text { Waist circumference (male) } \\
\text { Waist circumference (female) }\end{array}$ & $\begin{array}{l}1.854 \\
4.358\end{array}$ & $\begin{array}{l}0.833-4.120 \\
1.687-12.584\end{array}$ & $\begin{array}{c}0.332 \\
<0.005\end{array}$ & $\operatorname{HbAlc}(8-10 \%)$ & 1.254 & $0.951-2.567$ & 4.512 & $1.452-13.985$ \\
\hline Cholesterol & 1.450 & $0.858-2.246$ & 0.323 & Waist circumference & 2.156 & $1.012-5.125$ & 4.875 & $1.802-13.115$ \\
\hline $\begin{array}{l}\text { Triglyceride } \\
\text { Smoking }\end{array}$ & 1.086 & $\begin{array}{l}0.665-1.786 \\
0568-1956\end{array}$ & $\begin{array}{l}0.210 \\
0.041\end{array}$ & Duration of diabetes & 2.785 & $1.156-3.759$ & 2.568 & $1.702-3.778$ \\
\hline $\begin{array}{l}\text { Smoking } \\
\text { Duration of diabetes }\end{array}$ & $\begin{array}{l}0.856 \\
2.596\end{array}$ & $\begin{array}{l}0.568-1.956 \\
1.236-3.569\end{array}$ & $\begin{array}{c}0.041 \\
<0.005\end{array}$ & Systolic blood pressure & 2.889 & $1.852-6.854$ & 1.023 & $0.858-2.568$ \\
\hline Systolic blood pressure & 1.998 & $1.223-3.254$ & $<0.005$ & I: confidence interval. $\mathrm{HbAl}$ & noglobin. & & & \\
\hline Diastolic blood pressure & 1.235 & $0.847-2.547$ & 0.210 & & & & & \\
\hline
\end{tabular}

- 321 patients participated in the study. 163 women (50.78\%). Type 1 diabetes 19 patients (8.6\%).

- The mean age $58.21 \pm 11.87$ years. Mean diabetes duration $8.19 \pm 6.96$

- Mean HbA1c $8.2 \pm 2.1 \%$.

- Prevalence of MA $40.81 \%$, while the overt diabetic nephropathy was present in $2.8 \%$ of cases.

- 9 / 27 patients $(33.3 \%)$ with newly diagnosed diabetes presented already MA.

Diabetic nephropathy is a common chronic complication of diabetes mellitus.

Its early detection, and an aggressive treatment of different risk factors may prevent its progression. The search for the presence of MicroAlbuminuria might be part of the initial assessment for each person with type 2 diabetes. 